\title{
A generalization of Sylvester's and Frobenius' problems on numerical semigroups
}

\author{
by \\ ZDZISEAW SKUPIEŃ (Kraków)
}

1. Introduction. Our aim is to formulate and study a "modular change problem". Let $\mathcal{A}$ be a set of $t$ natural numbers $a_{1}, \ldots, a_{t}$ (which are coin denominations or semigroup generators). Integer linear combinations of these numbers are clearly multiples of gcd $\mathcal{A}$, their greatest common divisor. If indeterminate coefficients, say $x_{i}$ 's, are nonnegative, $x_{i} \in \mathbb{N}_{0}$, then those combinations form a numerical semigroup $S$ (under addition),

$$
S=S(\mathcal{A}):=\left\{n \in \mathbb{N}_{0} \mid n=\sum_{i=1}^{t} x_{i} a_{i}, \text { all } x_{i} \in \mathbb{N}_{0}\right\},
$$

which includes 0 and all multiples of $\operatorname{gcd} \mathcal{A}$ large enough. In fact, the following is known.

Proposition 1.1. All integer linear combinations of integers $a_{i}$ in $\mathcal{A}$ coincide with all the multiples of $\operatorname{gcd} \mathcal{A}$. If the coefficients are nonnegative integers, the combinations include all multiples of $\operatorname{gcd} \mathcal{A}$ large enough.

Let $\Omega(=\Omega(\mathcal{A})=|\mathbb{N}-S| \leq \infty)$ denote the cardinality of the complement of $S$ in $\mathbb{N}$. Hence, if the given numbers are relatively prime, that is,

$$
\operatorname{gcd}\left(a_{1}, \ldots, a_{t}\right)=1
$$

then $\Omega<\infty$ is the number of integers $n \in \mathbb{N}_{0}$ without any representation

$$
n=\sum_{i=1}^{t} x_{i} a_{i},
$$

with

$$
\text { all } x_{i} \in \mathbb{N}_{0} \text {. }
$$

The largest of these omitted $n$ 's is denoted by $g(\mathcal{A})$ (or $N(\mathcal{A})$ ); by definition $g(\mathcal{A})=\infty$ if $\Omega=\infty$, and $g(\mathcal{A})=-1$ if $\Omega=0$. The study of the functions $\Omega$ and $g$ dates back to Sylvester [14] and Frobenius (cf. [2]), respectively. Another related function - the number of partitions (1.2)-(1.3) 
of $n$, denoted by $\nu_{n}(\mathcal{A})$-is older and was studied by Euler. The study of $\Omega, g$, and/or $\nu_{n}$ constitutes the classical "change problem" (cf. [9], where only $\nu_{n}$ is considered).

Let $q \in \mathbb{N}$ and let $L, L=L_{q}$, be a complete system of residues modulo $q$ (e.g., $\mathbb{Z} \supset L=\{0,1, \ldots, q-1\}$ unless otherwise stated). For a $\kappa \in L$, we impose the additional requirement

$$
\sum_{i=1}^{t} x_{i} \equiv \kappa(\bmod q)
$$

and consider the related functions $\Omega_{\kappa}, N_{\kappa}$ and $\nu_{n \kappa}$ which represent the number of so-called $\kappa$-omitted integers $n$ (among nonnegative ones, $n \in \mathbb{N}_{0}$ ); the largest of them, $+\infty$, or -1 ; and the number of $\kappa$-representations of $n$, respectively. Then $(\mathcal{A}, q)$ is the pair of arguments of the functions and

$$
g(\mathcal{A}, q):=\max \left\{N_{\kappa}(\mathcal{A}, q): \kappa \in L_{q}\right\} .
$$

This new problem, the "modular change problem", includes the classical one (for $q=1$ ) and is prompted by applications of the problem (1.2)-(1.4) in constructive graph theory [13] where the following condition is desirable.

$$
\text { A solution exists for all natural } n \text { large enough. }
$$

Our main result yields a useful equivalent of the condition (1.5) (or finiteness of $g$ ) in case of our modular problem. Moreover, explicit formulae in case of two generators $(t=2)$ and, in general case, efficient algorithms for evaluating both all $\Omega_{\kappa}$ and all $N_{\kappa}$ are provided.

THEOREM 1.2. The finiteness of an $N_{\kappa}(\mathcal{A}, q)$ is equivalent to the conjunction of (1.1) and

$$
\operatorname{gcd}\left(q, a_{2}-a_{1}, a_{3}-a_{2}, \ldots, a_{t}-a_{t-1}\right)=1,
$$

and is equivalent to the finiteness of $g$ ( or all $N_{\kappa}$ 's).

The proof of necessity uses the general solution of a linear Diophantine equation. (It is not excluded that $t=1$, in which case (1.1) and (1.6) mean that $a_{1}=1=q$.)

A correct reference to Sylvester's problem (and result, proved by W. J. C. Sharp [14] using a generating function) will be provided.

2. General results. We need the following notation:

$$
D_{i}=\operatorname{gcd}\left(a_{1}, \ldots, a_{i}\right), \quad D_{0}:=0,
$$

whence $D_{1}=a_{1}$ and $D_{i}=\operatorname{gcd}\left(D_{i-1}, a_{i}\right), i=1, \ldots, t$. It is known that the 
general integer solution $x$ of (1.2) is the integer vector

$$
x=\widetilde{x}_{0}+\sum_{j=1}^{t-1} u_{j} y_{j}
$$

where $\widetilde{x}_{0}$ is a particular integer solution of (1.2) and $y_{j}$ 's are $t-1$ integer vectors which form a basis for the rational solution space of the simplified (homogeneous) equation

$$
\sum_{i=1}^{t} x_{i} a_{i}=0
$$

such that $u_{j}$ can be arbitrary integers. Hence, each $y_{j}$ is a $t$-vector which is divisor minimal, that is, its components are relatively prime. In particular, it is known that a solution $y$ of $(2.1)$ for $t=2, y=\left(x_{1}, x_{2}\right)$, is unique up to a factor of \pm 1 ,

$$
y= \pm\left(a_{2} / D_{2},-a_{1} / D_{2}\right) .
$$

For $j=1, \ldots, t$, let $\xi_{j}$ be an integer column $j$-vector with components $\xi_{i j}$ satisfying the auxiliary equation

$$
\sum_{i=1}^{j} a_{i} \xi_{i j}=D_{j}
$$

whence $\xi_{1}=\xi_{11}=1$. Assume that not only all $\xi_{j}$ but also $\widetilde{x}_{0}$ and all $y_{j}$ are column vectors, $y_{j}=\left[y_{i j}\right]_{t \times 1}$. Then

$$
\widetilde{x}_{0}=n \xi_{t} / D_{t}
$$

provided that $D_{t} \mid n$. By Proposition 1.1, the equation (2.3) can be replaced by

$$
D_{j-1} w_{j}+a_{j} \xi_{j j}=D_{j} \quad(j=1, \ldots, t) .
$$

Now, a solution of (2.4) determines the last component $\xi_{j j}$ of the vector $\xi_{j}$ and the remaining components can be computed recursively,

$$
\xi_{i j}=\xi_{i, j-1} w_{j} \quad \text { for } i<j \text { and } j \geq 2 .
$$

We are now ready to construct all vectors $y_{j}, j<t$. Assume that the last $t-j-1$ components of $y_{j}$ are zero, and the $(j+1)$ th component $y_{j+1, j}$ is negative and has the smallest possible absolute value. Then

$$
D_{j} z_{j}+a_{j+1} y_{j+1, j}=0 \quad \text { for some } z_{j} \in \mathbb{N}_{0},
$$

whence, using (2.3), (2.2), and the Kronecker $\delta$ symbol, we finally have

$$
y_{j}=\left[\begin{array}{c}
z_{j} \xi_{j} \\
y_{j+1, j} \\
0
\end{array}\right]=\left(a_{j+1}\left[\begin{array}{c}
\xi_{j} \\
0
\end{array}\right]-D_{j}\left[\delta_{i, j+1}\right]_{t \times 1}\right) / D_{j+1} \quad(1 \leq j<t)
$$


The above method which produces a "first-column-missing upper triangular" matrix $\left[y_{i j}\right]_{t \times(t-1)}$ (see also [1]) usually gives solution vectors $y_{j}$ with large components $y_{i j}$ (in absolute value) depending on the ordering of $a_{i}$ 's. A computationally efficient method to find $D_{t}$ and a vector $\xi_{t}$ together with all basis solutions $y_{j}$ (with components small enough) can be found in $[6$, $5]$. The above method, however, readily gives the general solution to each equation (2.3). Namely, if $k$ replaces $j$ there, then $\widetilde{x}_{0}=\xi_{k}$ and the corresponding solution basis is formed by the columns of the leading $k \times(k-1)$ submatrix of $\left[y_{i j}\right]$.

From (2.5), using (2.3) to eliminate $\xi_{j j}$, we get

$$
\begin{aligned}
\sum_{i=1}^{t} y_{i j} & =\left(\xi_{j j} a_{j+1}-D_{j}+a_{j+1} \sum_{i=1}^{j-1} \xi_{i j}\right) / D_{j+1} \\
& =\left(D_{j}\left(a_{j+1}-a_{j}\right)+a_{j+1} \sum_{i=1}^{j-1}\left(a_{j}-a_{i}\right) \xi_{i j}\right) / a_{j} D_{j+1}, \quad j<t .
\end{aligned}
$$

Proof of Theorem 1.2. First, by Proposition 1.1, the existence of an integer solution of (1.2) for any $n$ is equivalent to (1.1).

Necessity of (1.1) is thus proved. Hence, if $p$ is a prime divisor of the left-hand side of (1.6) then $p \nmid a_{k}$ for all $k$ and therefore $p \mid \sum_{i} y_{i j}$ in (2.6) for all $j$. Then by $(2.0)$, for any $n=(k q-1+\kappa) a_{1}(k \in \mathbb{N})$ in (1.2), (1.4) is not satisfied since $p \mid q$, a contradiction.

Sufficiency. Using (2.0) and (2.6) one can see that (1.1) and (1.6) imply the existence of a solution to (1.2) and (1.4) for any $n$ and for any $\kappa \in L_{q}$. Now, let $-Y_{n, \kappa}$ and $Z_{n, \kappa}$ be the corresponding parts of the right-hand side of (1.2) with nonpositive and nonnegative coefficients, respectively. Assume that the number $+Y_{n, \kappa}$ is as small as possible. Thus $Y_{0,0}=0=Z_{0,0}$ (where $n=0$ and $\kappa=0$ ).

Let $-Y^{0}$ be a linear combination of $a_{i}$ 's such that, for all $i$, the coefficient of $a_{i}$ is chosen to be the smallest of (nonpositive) coefficients of the $a_{i}$ in all $-Y_{0, \kappa}($ where $n=0)$. For $n=1$ and $\kappa=0$, let $Y=Y_{1,0}$ and $Z=Z_{1,0}$ whence $1=-Y+Z$. Consider the following $a_{1}$ consecutive integers $n$ :

$$
\begin{gathered}
\left(a_{1}-1\right) Y+\quad Y^{0}, \\
\left(a_{1}-2\right) Y+Z+Y^{0}, \\
\ldots \ldots \ldots \ldots \ldots \\
\quad\left(a_{1}-1\right) Z+Y^{0} .
\end{gathered}
$$

Each of them is fully representable, i.e., has representations (1.2)-(1.4) for all $\kappa \in L_{q}$, because any representation can be modified by adding any of the $q$ expressions $0=-Y_{0, \kappa}+Z_{0, \kappa}$ where $n=Y^{0}-Y_{0, \kappa}$ has a representation (1.2) and (1.3) by the very definition of $Y^{0}$. Each larger integer also has full 
representations, by adding a multiple of $a_{1}$ to representations of one of the $a_{1}$ integers above.

The above sufficiency proof extends that of the existence of $g$ for $q=1$, due to Ö. Beyer, as presented in Selmer [12] (1986).

In what follows (1.1) and (1.6) are assumed. Moreover,

$$
a_{1}<\ldots<a_{t} .
$$

A generator which has a 1-representation (modulo $q$ ) by the remaining generators can be removed from $\mathcal{A}$ without altering the value of any $N_{\kappa}$. Call the set $\mathcal{A}$ of generators $q$-independent if either $q=1=t=a_{1}$ or $t>1$ and no $a_{i}$ in $\mathcal{A}$ is 1-representable modulo $q$ by the remaining generators; otherwise $\mathcal{A}$ is called $q$-dependent (1-representable modulo 1 means representable). Hence the 1-independence of $\mathcal{A}(q=1)$ is the known notion of independence of generators.

Note that

$$
|\mathcal{A}|=t \leq q a_{1}=q \min \mathcal{A}
$$

is a necessary condition for $\mathcal{A}$ to be $q$-independent (whence $a_{t} \geq\lceil t / q\rceil+t-1$ if $\mathcal{A}$ is $q$-independent).

In fact, suppose $q a_{1}<t$. Then $\left|\mathcal{A}-\left\{a_{1}\right\}\right| \geq q a_{1}$. Hence there is $j \geq 2$ such that $a_{j} \equiv a_{1}\left(\bmod q a_{1}\right)$ or there are $i, j \geq 2$ with $a_{i} \equiv a_{j}\left(\bmod q a_{1}\right)$. In either case $\mathcal{A}$ is $q$-dependent.

Recall that $g(\mathcal{A}, q)$ is the largest integer (or $+\infty$ ) which is not fully representable modulo $q$ by $\mathcal{A}$. The Frobenius problem consists in finding (an upper bound for) the integer $g(\mathcal{A}), g(\mathcal{A})=g(\mathcal{A}, 1)=N_{0}(\mathcal{A}, 1)$, i.e., if $q=1$ and $\kappa=0$. In this context we shall assume

$$
a_{t} \leq g\left(\mathcal{A}-\left\{a_{t}\right\}, q\right) \quad \text { if } t \geq 2,
$$

i.e., first we shall possibly eliminate excessively large (irrelevant) generators. This natural assumption, which only admits of independence of the largest generator $a_{t}$ from the remaining ones, is usually omitted in the published upper bounds for $g(\mathcal{A}, 1)$ or - as in [11] — it is sometimes replaced by requiring the independence of the whole $\mathcal{A}$.

Given a positive integer $\widetilde{n}$ which has a representation (1.2)-(1.3) with $n=\widetilde{n}$ (e.g., $\widetilde{n}=a_{i}, \sum a_{i}$, etc., the smallest $\widetilde{n}=a_{1}$ ), let

$$
m=q \widetilde{n}
$$

and, for each residue $r$ modulo $m$ and a fixed $\kappa \in L_{q}$, let $n_{r \kappa}$ be the least $n$ which is in the residue class of $r$ modulo $m$ and has a $\kappa$-representation. Hence, by the choice of $m$, if $n \equiv r(\bmod m), n$ clearly has a $\kappa$-representation if and only if $n \geq n_{\kappa \kappa}$. Thus, the finiteness of $N_{\kappa}$ 's is equivalent to the 
existence of all numbers $n_{r \kappa}$; moreover,

$$
N_{\kappa}=\max _{r} n_{r \kappa}-m
$$

because, if $N_{\kappa}$ is finite, there is $\varrho \in \mathbb{N}_{0}$ with $\varrho<m$ such that $N_{\kappa} \equiv \varrho$ $(\bmod m)$, whence $N_{\kappa}$ is clearly $m$ smaller than $n_{\varrho \kappa}$. This extends a formula for $g$ due to Brauer and Shockley [2, Lemma 3] $(q=1$ and $\kappa=0)$. Thus, knowing the $q m$ numbers $n_{r \kappa}$ [and a $\kappa$-representation of each $n_{r \kappa}$ ] we can determine all sets, say $\mathfrak{I}_{\kappa}^{\mathrm{c}}$, of $\kappa$-omitted integers [and a $\kappa$-representation of each positive $n$ such that $\left.n \notin \mathfrak{I}_{\kappa}^{\mathrm{c}}\right]$. Analogously, on partitioning $\mathfrak{I}_{\kappa}^{\mathrm{c}}$ into residue classes modulo $m$,

$$
\begin{array}{rlr}
\Omega_{\kappa}:=\left|\mathfrak{I}_{\kappa}^{\mathrm{c}}\right| & =\sum_{r=0}^{m-1}\left(n_{r \kappa}-r\right) / m \\
& =-(m-1) / 2+\sum_{r} n_{r \kappa} / m \quad \text { (cf. [11]) } \\
& =\sum_{r}\left\lfloor n_{r \kappa} / m\right\rfloor
\end{array}
$$

This formula generalizes those by Selmer [11, Theorem] and Nijenhuis [7], respectively, for $\Omega$ if $q=1$.

3. The case of two generators, $t=2$. Throughout this section,

$$
\kappa \in\{-1,0, \ldots, q-2\} .
$$

Let us use standard notation:

$$
a=a_{1}, \quad b=a_{2}, \quad x=x_{1}, \quad y=x_{2} \quad(a<b) .
$$

Since (1.1) and (1.6) are assumed to hold,

$$
\operatorname{gcd}(a, b)=1=\operatorname{gcd}(q, b-a) \cdot
$$

Sylvester's contribution to the change problem is misquoted or misplaced quite often (cp. [8, 11, 12, 4] and (!) [13]). The following is what Sylvester actually presents in [14] (where in fact $p$ and $q$ stand for $a$ and $b$, resp.): "If $a$ and $b$ are relative primes, prove that the number of integers inferior to $a b$ which cannot be resolved into parts (zeros admissible), multiples respectively of $a$ and $b$, is

$$
\frac{1}{2}(a-1)(b-1) . "
$$

It is explained in [14] by means of an example that integers in question are to be positive. Notice that it belongs to the mathematical folklore now that the bound $a b$ above [integer $a b-a-b$ ] is the largest integer which is not representable as a linear combination of $a$ and $b$ with positive [nonnegative] integer coefficients. 
We refer to $\kappa$-representations, $\kappa$-omitted integers and symbols $g(\mathcal{A}, q)$ and $N_{\kappa}(\mathcal{A}, q)$ as defined in Introduction. In order to avoid trivialities, assume

$$
1 \leq a<b \quad \text { but } \quad a>1 \quad \text { if } q=1,
$$

because if $1 \in \mathcal{A}$ then $S=\mathbb{N}_{0}$, whence $g(\{1, b\}, q)=-1$ if $q=1$. Define

$$
g:=q a b-a-b,
$$

whence, by (3.2), $g$ is odd;

$$
\begin{aligned}
N_{\kappa} & :=q a b-b-(q-1-\kappa) a, & & -1 \leq \kappa \leq q-2 \\
& =g-(q-2-\kappa) a, & & \text { by }(3.4) .
\end{aligned}
$$

TheOREM 3.1. Under the above assumptions, if $t=2$ and $\mathcal{A}=\{a, b\}$, the largest $\kappa$-omitted integer $N_{\kappa}(\mathcal{A}, q)=N_{\kappa}\left(\right.$ whence $\left.g(\mathcal{A}, q)=N_{q-2}=g\right)$ and $\Omega_{\kappa}=(g+1) / 2$ is the number of $\kappa$-omitted integers.

Hence the interval $[0, g]$ contains as many $\kappa$-representable integers as $\kappa$-omitted ones. The proof is based on a series of auxiliary results which follow.

Proposition 3.2 (Folklore). If $a, b \in \mathbb{N}$ and $\operatorname{gcd}(a, b)=1$ then, for each $n \geq(a-1)(b-1)$, there is exactly one pair of nonnegative integers $\varrho$ and $\sigma$ such that $\sigma<a$ and $n=\varrho a+\sigma b$.

Notice for the proof that, for $j=0,1, \ldots, a-1$, if $\operatorname{gcd}(a, b)=1$, all integers $n-j b$ are mutually distinct modulo $a$. Hence, for exactly one $j$, say $j=\sigma$, we have $n=\varrho a+\sigma b$, whence $\varrho \geq 0$ because $\varrho a \geq-a+1$.

It is well known that

$$
(x, y)=\left(x^{0}+u b, y^{0}-u a\right), \quad u \in \mathbb{Z},
$$

is a general solution of (1.2) in our case, which agrees with (2.0) and (2.2). Hence we have

Proposition 3.3. For any $\kappa$, if $n<q a b$ (or $n \leq g$ in (3.4)) then $n$ has at most one $\kappa$-representation.

Using (3.4), let

$$
\mathfrak{I}:=\mathbb{Z} \cap[0, g], \quad \mathfrak{I}^{\prime}:=\mathbb{Z} \cap[0, q a b) .
$$

Let $\mathfrak{I}_{\kappa}$ denote the set of $\kappa$-representable integers and let

$$
\mathfrak{I}_{\kappa}:=\mathfrak{I}_{\kappa} \cap \mathfrak{I}, \quad \mathfrak{I}_{\kappa}^{\prime}:=\mathfrak{I}_{\kappa} \cap \mathfrak{I}^{\prime}, \quad \mathfrak{I}_{\kappa}^{\mathrm{c}}:=\mathfrak{I}-\mathfrak{I}_{\kappa} .
$$

Moreover, $k+A:=\{k+x \mid x \in A\}$ if $A \subseteq \mathbb{Z}$. Notice that if $q=1$ (and $\kappa=-1$ ), then $\mathfrak{I}_{\kappa}=S$, whence, by Proposition 3.2 and formula (3.4), $\mathfrak{I}_{\kappa}^{\mathrm{c}}=\mathbb{N}_{0}-S$. We are going to show that in general $\mathfrak{I}_{\kappa}^{\mathrm{c}}$ is the set of $\kappa$-omitted integers (cf. the end of the preceding section). 
Proposition 3.4. For any $\kappa, N_{\kappa} \in \mathfrak{I}_{\kappa}^{\mathrm{c}}$.

Proof. By (3.3) and (3.5), $N_{\kappa} \geq 0$. By (3.5) and (3.6), all solutions of (1.2) for $n=N_{\kappa}$ are of the form

$$
x=\kappa+1+(q-u) b-q \quad \text { and } \quad y=u a-1, \quad u \in \mathbb{Z} .
$$

Then $x, y \geq 0$ can be satisfied only if $1 \leq u<q$, which is a contradiction if $q=1$; otherwise, due to $(3.2), x+y(=\kappa+(b-1) q-(b-a) u) \not \equiv \kappa(\bmod q)$, contrary to (1.4).

The following transformation is used by Nijenhuis and Wilf [8] in order to solve Sylvester's problem (with $q=1$ and $\kappa=-1$ ).

Proposition 3.5. The transformation

$$
\varphi: \mathfrak{I}_{\kappa} \ni n \mapsto g-n
$$

is a bijection onto $\mathfrak{I}_{q-2-\kappa}^{\mathrm{c}}$ if $0 \leq \kappa \leq q-2$, and onto $\mathfrak{I}_{\kappa}^{\mathrm{c}}$ if $\kappa=-1$.

Proof. By (3.4) and (3.5), $g=N_{q-2}$. Hence, if $n \in \mathfrak{I}_{\kappa}$ then $\varphi(n) \notin$ $\mathfrak{I}_{q-2-\kappa}$ because otherwise $g=n+\varphi(n) \in \mathfrak{I}_{q-2}$, contrary to Proposition 3.4. Moreover, injectivity of $\varphi$ is clear. Notice that assumptions (3.2) ensure the existence of a solution $\left(x_{1}, y_{1}\right)$ of (1.2) such that $0 \leq x_{1}<q b$ and $x_{1}+y_{1} \equiv q-2-\kappa(\bmod q)$. Suppose $n \in \mathfrak{I}_{q-2-\kappa}^{\mathrm{c}}$ if $\kappa \geq 0$, and $n \in \mathfrak{I}_{-1}^{\mathrm{c}}$ if $\kappa=-1$. Then clearly $y_{1}<0$. Therefore, by (3.4), $g-n=\left(q b-1-x_{1}\right) a+$ $\left(-y_{1}-1\right) b \in \mathfrak{I}_{\kappa}$, whence $\varphi(g-n)=n$, which proves surjectivity of $\varphi$.

COROLlary 3.6. $\left|\mathfrak{I}_{-1}\right|=\left|\mathfrak{I}_{-1}^{\mathrm{c}}\right|=|\mathfrak{I}| / 2=(g+1) / 2$ (cf. (3.7)).

PROPOSITION 3.7.

$$
(q-2-\kappa) a=\min \begin{cases}\mathfrak{I}_{q-2-\kappa} & \text { if } \kappa \geq 0, \\ \mathfrak{I}_{-1} & \text { if } \kappa=-1 .\end{cases}
$$

Proposition 3.8. $\max \left(\mathbb{Z}-\mathfrak{I}_{\kappa}\right)=N_{\kappa}$.

Proof. Owing to Proposition 3.4, it is enough to show that $k \in \mathfrak{I}_{\kappa} \rightarrow$ if $k>N_{\kappa}$. To this end, assume $q \geq 2$ because the case $q=1$ is covered by Proposition 3.2. Next, assume $\kappa \neq q-2$ and $N_{\kappa}<k \leq g$. Then, by (3.5), $0 \leq g-k<g-N_{\kappa}=(q-2-\kappa) a$, whence, due to Propositions 3.7 and 3.5, $k \in \mathfrak{I}_{\kappa}$ and we are done. Finally, assume that $n=k>g\left(=N_{q-2}\right)$. Then

$$
n_{k}:=k-(q-1) a b \geq(a-1)(b-1) \quad \text { by }(3.4),
$$

whence, by Proposition 3.2, $n_{k}=\varrho a+\sigma b$ for exactly one pair $(\varrho, \sigma) \geq(0,0)$ and $\sigma<a$. Hence, (1.2) and $x, y \in \mathbb{N}_{0}$ are satisfied if

$$
x=\varrho+(q-1-j) b \quad \text { and } \quad y=\sigma+j a
$$

for $q$ consecutive values of $j, j=0, \ldots, q-1$, whence, by (3.2), the congruence (1.4) is satisfied for one of these $j$ 's. Thus $k \in \mathfrak{I}_{\kappa}$.

COROLlary 3.9. $\mathfrak{I}_{\kappa}^{\mathrm{c}}$ is the set of $\kappa$-omitted integers. 
Proof of Theorem 3.1. The first part of the Theorem follows from Proposition 3.8. As for the counting part, let

$$
\mathfrak{I}_{\kappa}^{-}=\mathfrak{I}_{\kappa}-\{g, g-1, \ldots, g-a+1\} .
$$

Then, by (3.7), Proposition 3.8 and formula (3.5), $\left|\mathfrak{I}_{\kappa}^{-}\right|=\left|\mathfrak{I}_{\kappa}\right|-a$ for $\kappa<$ $q-2$. Moreover, using Proposition 3.3, one can see that, for each $\kappa \geq 0$,

$$
\psi_{\kappa}: \mathfrak{I}_{\kappa-1}^{-} \ni n \mapsto n+a
$$

is a bijection onto $\mathfrak{I}_{\kappa}-\{(k q+\kappa) b \mid k=0,1, \ldots, a-1\}$, a set of cardinality $\left|\mathfrak{I}_{\kappa}\right|-a$, by (3.7), (3.4) and (3.1). Hence, $\left|\mathfrak{I}_{\kappa-1}\right|=\left|\mathfrak{I}_{\kappa}\right|$ for each $\kappa \geq 0$, which, due to (3.7) and Corollaries 3.6 and 3.9, ends the proof.

The following result extends Corollary 3.9 and Proposition 3.3 and reduces determining $\nu_{n \kappa}$, the number of $\kappa$-representations of $n$, to the membership problem for the residue $(n \bmod q a b)$ (cf. [9] for $q=1)$.

COROLlary 3.10. (A) The set of integers $n$ such that $n \in \mathbb{N}_{0}$ and $\nu_{n \kappa}=$ $k, k \in \mathbb{N}_{0}$, is $\mathfrak{I}_{\kappa}^{\mathrm{c}}$ of cardinality $(g+1) / 2$ if $k=0$, else $\left((k-1) q a b+\mathfrak{I}_{\kappa}^{\prime}\right) \cup$ $\left(k q a b+\mathfrak{I}_{\kappa}^{\mathrm{c}}\right)$ of cardinality qab. Hence, kqab $+\mathfrak{I}_{\kappa}$ is the set of integers $n$ such that $\nu_{n \kappa} \geq k+1, k \geq 0$. Moreover,

(B) For $n \in \mathbb{N}_{0}, \nu_{n \kappa}$ is $\lfloor n /(q a b)\rfloor+1$ or $\lfloor n /(q a b)\rfloor$ according as ( $n \bmod$ qab) is representable $\left(\in \mathfrak{I}_{\kappa}\right)$ or is not $\left(\in \mathfrak{I}_{\kappa}^{\mathrm{c}}\right)$.

Theorem 3.1 is equivalent to a part of the next result. Moreover, the author's paper [13] referred to above contains a result equivalent to the non-counting parts of this result in case $q=2$ and $\kappa=-1$.

THEOREM 3.11. Given any integers $m_{a}, m_{b}$ and

$$
\widetilde{n}:=a m_{a}+b m_{b}, \quad \widetilde{N}_{\kappa}:=\widetilde{n}+g-\left(q-1-\widetilde{\varepsilon}_{\kappa}\right) a \quad(=\widetilde{n}+g \text { if } q=1)
$$

(see (3.4) for $g$ ) where

$$
\widetilde{\varepsilon}_{\kappa} \equiv\left(\kappa+1-m_{a}-m_{b}\right)(\bmod q), \quad 0 \leq \widetilde{\varepsilon}_{\kappa}<q,
$$

all integers $n, n \geq \widetilde{n}$, which cannot be represented as integer linear combinations $x a+y b$ under assumptions (3.2) and (3.3) and requirements $x \geq m_{a}$, $y \geq m_{b}$ and $x+y \equiv \kappa(\bmod q)$ are in the interval $\left[\widetilde{n}, \widetilde{N}_{\kappa}\right]$, their number is $(g+1) / 2$ (which is independent of $\kappa)$ and $\widetilde{N}_{\kappa}$ is the largest of them. On the other hand, the uniqueness of $(x, y)$ is implied by either of the following inequalities: $m_{a} \leq x<m_{a}+q b, m_{b} \leq y<m_{b}+q a$.

4. Algorithms. Let $g(\mathcal{A}, q)<\infty$ and $t>1$. Then two algorithms for evaluating the integers $N_{\kappa}$ and $\Omega_{\kappa}$ can be presented. One, $(\mathrm{W})$ : a toroidal lattice-of-lights, extends Wilf's circle-of-lights [15], and another one, (N): a minimum-path algorithm, devised after Nijenhuis' [7]. 
The algorithm (W) processes consecutive integers $n \in \mathbb{N}_{0}$ using the following simple rule. $(n=) 0$ is 0-representable; any $n \in \mathbb{N}$ is $(\kappa+1)$ representable iff $n-a_{i}$ is $\kappa$-representable for some $i=1,2, \ldots, t$ where $\kappa \in L_{q}$. The corresponding information (0: no (or light off) or 1: yes (light on)) on $n$ and any $\kappa$ is put at position $(r, \kappa), r=\left(n \bmod a_{t}\right)$, of the resulting doubly cyclic (toroidal) $0-1$ list of size $q a_{t}$. Additionally, $\operatorname{RP}[\kappa]$, the number of $\kappa$-representable integers, is updated and the $a_{1}$ th of consecutive $\kappa$-representable integers $n$ is recorded as $N[\kappa]$. The process stops at the first $n$ which is the $a_{1}$ th of consecutive fully representable integers. Then output is $N_{\kappa}=N[\kappa]-a_{1}$ and $\Omega_{\kappa}=n+1-\operatorname{RP}[\kappa]$. Thus, since $t \leq a_{t}$, space complexity is $O\left(q a_{t}\right)$. Since $g \geq a_{1}-1$, time complexity can be shown to be $O(t q g)$ or $O((t+q) g)$ depending on the (data structure dealing with $0-1$ vectors and) implementation. As a by-product the algorithm gives the following inequality which is not sharp in general but, for $q=1$, it improves on one due to Wilf:

$$
g \leq\left(q a_{t}-2\right) a_{t}-1 \quad \text { for } t \geq 2 .
$$

Proof. This is true if $t=2$ (and $q=1$ ). Else, if not all lights are on, each full sweep around the lattice increases the number of lights which are on because otherwise (it would only cause the rotation of lights and) $g$ would be infinite, contrary to Theorem 1.2. We may stop at $n$ such that at most $z:=\left\lceil a_{t} / a_{1}\right\rceil-1$ lights are left off. Then $g \leq n+z a_{1}$. Since 1 is at $(0,0)$ due to the initial condition, the first sweep adds at least two new 1's (if $t>2$ or $q>1$ ). Thus, $n \leq\left(q a_{t}-2-z\right) a_{t}$, whence the result follows.

The bound (4.1) on $g$ can be improved considerably. Erdös-Graham's important upper bound for $g(\mathcal{A}, 1)$ (see [3]) (whose simple proof can be found in Rödseth [10]) can be extended to any admissible $q$. Adapting Rödseth's argument to formula (2.10) with $m=q a_{t}$ gives the result. Let $q \mathcal{A}$ be the sum of $q$ copies of the set $\mathcal{A}$, let $\mathcal{A}_{0}=q \mathcal{A} \cup\{0\}-\left\{q a_{t}\right\}$, and let $h=2\left\lfloor a_{t} /(t-1+1 / q)\right\rfloor$. Then

$$
\begin{aligned}
N_{0}(\mathcal{A}, q) & \leq \max \sum_{b_{j} \in \mathcal{A}_{0}} y_{j} b_{j}-q a_{t} \\
& \begin{array}{l}
\text { with max over } y_{j} \text { 's from } \mathbb{N}_{0} \text { such } \\
\text { that } \sum y_{j} \leq h \text { and some of } y_{j} \text { 's } \\
\text { are small, }
\end{array} \\
& \leq \max _{x_{i} \in \mathbb{N}_{0}, \Sigma x_{i} \leq q h, x_{t}<q} \sum_{i=1}^{t} x_{i} a_{i}-q a_{t} \\
& \leq(q h-q+1) a_{t-1}-a_{t} \quad(\text { for } \kappa=0),
\end{aligned}
$$

and

$$
N_{\kappa}(\mathcal{A}, q) \leq N_{0}(\mathcal{A}, q)+\kappa a_{1}, \quad \kappa=0,1, \ldots, q-1,
$$


whence

$$
g(\mathcal{A}, q) \leq 2 q a_{t-1}\left\lfloor a_{t} /(t-1+1 / q)\right\rfloor-(q-1)\left(a_{t-1}-a_{1}\right)-a_{t} .
$$

Therefore $g$ is $O\left(q a_{t}^{2} / t\right)$ (and so is $\Omega_{\kappa}$ for any $\kappa$ because $\left.\Omega_{\kappa} \leq g+1\right)$. It can be seen that the bound (4.2) is sharp in the sense that, for each $q \geq 1$ and each $t \geq 2$, there is an $\mathcal{A}$ with $|\mathcal{A}|=t, a_{t}$ large enough and $g(\mathcal{A}, q)=\Theta\left(q a_{t}^{2} / t\right)$, $\Theta$ indicating the exact order of magnitude.

The algorithm $(\mathrm{N})$ is more efficient but is also only pseudo-polynomial (i.e., a common bound on complexities is a polynomial in $t, q$ and some $\left.a_{i}\right)$. The algorithm is based on generating all $q^{2} a_{1}$ integers $n_{r \kappa}$ as sums of generators $a_{i}$, see formulae (2.10)-(2.11) with $m=q a_{1}$, the smallest possible value of $m$. It maintains a heap (i.e., a binary tree) of $\kappa$-heaps whose entries are available sums which are put in increasing order along paths going from the root of the $\kappa$-heap, $\kappa$-heaps being similarly ordered by their roots. The algorithm starts by taking 0 as $n_{00}$. Next, if $n_{r \kappa}$ is identified (as the smallest available sum) and removed from the heap, the algorithm accommodates each of the sums $s=n_{r \kappa}+a_{j}$ in the $(\kappa+1)$-heap, i.e., inserts $s$ as the $(r, \kappa+1)$-entry where $r=(s \bmod m)$ provided that the entry either has not appeared yet or is larger than $s$. Time of labour associated with each $s$ is $O\left(\log _{2}\left(q^{2} a_{1}\right)\right)$. The space and time complexities of the algorithm are $O\left(t+q^{2} a_{1}\right)$ and $O\left(t q^{2} a_{1} \log _{2}\left(q^{2} a_{1}\right)\right)$, respectively. Our complexity estimates correct some of those by Nijenhuis [7].

For the set $\mathcal{A}=\{271,277,281,283\}$ (dealt with by Wilf [15] for $q=1$ ), our computer programs $(\mathrm{W})$ and $(\mathrm{N})$ found data presented in Table 1 for $q=5,3,1$ in stated seconds on PC AT $386(20 \mathrm{MHz})(\mathrm{A})$ and XT (8 MHz) (X), respectively. Notice that $q=2$ (or any even $q$ ) is not allowed.

Table 1

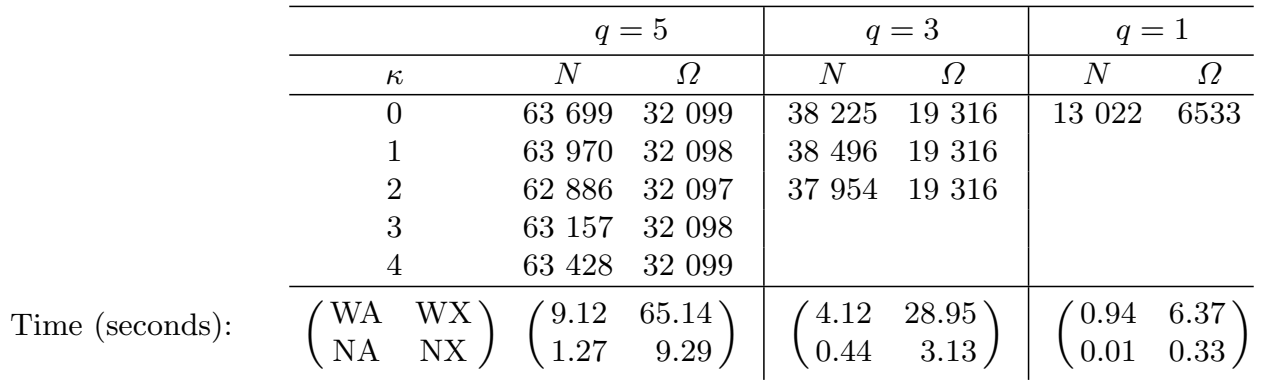

Programs (N) and (W) can easily be supplemented so as to generate $q^{2} a_{1}$ integers $n_{r \kappa}^{(1)}$ (this is the smallest $\kappa$-representable integer in the residue class of $r$ modulo $q a_{1}$ ), together with an explicit representation of each of them. This can yield all sets $\mathfrak{I}_{\kappa}^{\mathrm{c}}$ of omitted integers [and some representations of the remaining ones]. 
5. Problems and concluding remarks. A natural, though not easy, problem is to study the function $\kappa \mapsto\left(N_{\kappa}, \Omega_{\kappa}\right)$ in case $t \geq 3$. Partial questions can be of interest.

(a) Formulae (3.5) in case $t=2$ and many examples of pairs $(\mathcal{A}, q)$ with $t \geq 3$ suggest that $N_{\kappa} \in\left\{g-j a_{1} \mid j=0,1, \ldots, q-1\right\}, g=g(\mathcal{A}, q)$. Nevertheless, this is not the case in general. Namely, if $a$ and $b$ are relatively prime natural numbers, $a<b$ and $b-a$ is odd then, for $\mathcal{A}=\{a, b, a+b\}$ and $q=2$, one has $g=g(\mathcal{A}, 2)=a b-a=N_{b \bmod 2}$ and $a b / 2=\Omega_{\kappa}$ for both $\kappa=0,1$; moreover,

$$
N_{a \bmod 2}= \begin{cases}g+a-b=a b-b & \text { if } b<2 a, \\ g-a & \text { otherwise. }\end{cases}
$$

(For the proof, use representations by the set $\{a, b\}$ with $q=1$, see Section 3 . In particular, all omitted integers there and half of the set $\{i a, j b \mid i=$ $0, \ldots, b-1 ; j=1, \ldots, a-1\}$ can coincide with our $\kappa$-omitted integers.) It is easily seen, however, that all $N_{\kappa}$ 's are in the closed interval $\left[g-(q-1) a_{1}, g\right]$. In fact, use (2.7) and (2.10) with $m=q a_{1}$ to see that all integers $n_{r \kappa}+a_{1}$ are $(\kappa+1)$-representable and their residues modulo $q a_{1}$ form a complete system, whence

$$
N_{\kappa+1} \leq N_{\kappa}+a_{1} \quad \text { for all pairs } \kappa, \kappa+1 \text { in } \mathbb{Z} .
$$

Hence, the result follows.

(b) For $q=1$, it is known [8] that $\Omega \geq(g+1) / 2$. For any $q$, by using the transformation $n \mapsto g-n$ as in Proposition 3.5, one can prove $\max _{\kappa} \Omega_{\kappa} \geq(g+1) / 2$ or, more generally,

$$
\max _{\kappa} \Omega_{\kappa}+\min _{\kappa} \Omega_{\kappa} \geq g+1 .
$$

Characterize all (or find more interesting examples of) pairs $(\mathcal{A}, q)$ with $t \geq 3$ such that $\Omega_{\kappa}=$ const on $L_{q}(q>1)$ where possibly const $=(g+1) / 2$ $(q \geq 1)$ (cp. $t=2$ above or supersymmetric semigroups in [4] for $q=1$ ).

(c) Characterize $(\mathcal{A}, q)$ with $q>1$ and $t=|\mathcal{A}|>2$ such that $\Omega_{\kappa}>$ $g(\mathcal{A}, q) / 2$ for all $\kappa \in L_{q}$. Characterize $\mathcal{A}$ such that this holds for all admissible $q$ (or - on the contrary - does not hold for almost all such $q$ ). Determine the largest admissible integer $q$, denote it by $\xi(\mathcal{A})$, such that

$$
\Omega_{\kappa}>g(\mathcal{A}, q) / 2 \quad \text { for all } \kappa \in L_{q} .
$$

Let $\xi^{\prime}(\mathcal{A})$ be the largest integer $k$ such that (5.1) holds for all admissible $q \leq k$. Notice that $\xi^{\prime} \leq \xi$ for all $t \geq 2$. If $t=1$ then $\xi^{\prime}=\infty$ and $\xi=1$ (and $\mathcal{A}=\{1\})$. Characterize $\mathcal{A}$ with $\xi^{\prime}=\xi$.

In what follows, $\mathcal{A}=\mathcal{A}_{t, a}:=\{a, a+1, \ldots, a+t-1\}$ with $t \geq 2$, a set of consecutive generators (dealt with in [8]) with $t$ elements, $a$ being the 
smallest. One can see now that $\xi^{\prime}=\infty=\xi$ iff $t-1$ divides $a$, iff $\Omega_{\kappa}=$ const on $L_{q}$ for each $q$; moreover, const $=(g+1) / 2$ iff $a=1=q$ or $q=2$ and $t-1 \mid a-1$, or finally, $t-1 \mid a-2$ with the restriction that $q=1$ if $t \geq 4$. On the other hand, for $t \geq 3$, we have $\xi^{\prime}=t$ and $\xi=a$ if $t-1 \mid a-1$ unless $a=1$ and then $\xi^{\prime}=2=\xi$.

Acknowledgments. The author is indebted to his daughter Anna /Sliz of Toronto and Dr. Paul Vaderlind of Stockholm for providing him with copies of Sylvester's contribution [14(a)]. He also thanks Dr. Anna Rycerz for her calling the author's attention to Nijenhuis' paper [7]. Remarks of Prof. G. Hofmeister which resulted in improving the contents of the paper are gratefully acknowledged. Partial support of Polish KBN Grant Nr 2 P301 05003 is acknowledged.

\section{References}

[1] J. Bond, Calculating the general solution of a linear Diophantine equation, Amer. Math. Monthly 74 (1967), 955-957.

[2] A. Brauer and J. E. Shockley, On a problem of Frobenius, J. Reine Angew. Math. 211 (1962), 215-220.

[3] P. Erdős and R. L. Graham, On a linear diophantine problem of Frobenius, Acta Arith. 21 (1972), 399-408.

[4] R. Fröberg, C. Gottlieb and R. Häggkvist, On numerical semigroups, Semigroup Forum 35 (1987), 63-83.

[5] S. Kertzner, The linear diophantine equation, Amer. Math. Monthly 88 (1981), 200-203.

[6] S. Morito and H. M. Salkin, Finding the general solution of a linear diophantine equation, Fibonacci Quart. 17 (1979), 361-368.

[7] A. Nijenhuis, A minimal-path algorithm for the "money changing problem", Amer. Math. Monthly 86 (1979), 832-834.

[8] A. Nijenhuis and H. S. Wilf, Representations of integers by linear forms in nonnegative integers, J. Number Theory 4 (1972), 98-106.

[9] G. Pólya and G. Szegö, Aufgaben und Lehrsätze aus der Analysis I, Springer, 1925 [revised and enlarged: Problems and Theorems in Analysis I, Springer, 1978, pp. 174 and 180 [Problems I 9, I 26-27].

[10] Ö. J. Rödseth, Two remarks on linear forms in non-negative integers, Math. Scand. 51 (1982), 193-198.

[11] E. S. Selmer, On the linear diophantine problem of Frobenius, J. Reine Angew. Math. 293/294 (1977), 1-17.

[12] - The local postage stamp problem, Part 1: General theory, Ch. II; Part 3: Supplementary volume, Supplement to Ch. II; preprints, University of Bergen, 42 (1986) and 57 (1990), resp.

[13] Z. Skupień, Exponential constructions of some nonhamiltonian minima, in: Proc. 4th CS Sympos. on Combinat., Graphs and Complexity (held in Prachatice 1990), J. Nešetřil and M. Fiedler (eds.), Ann. Discrete Math. 51, Elsevier, 1992, 321-328. 
[14] J. J. Sylvester, [Problem] 7382 (and Solution by W. J. Curran Sharp), The Educational Times 37 (1884), 26; reprinted in (a): Mathematical Questions, with their Solutions, from the "Educ. Times", with Many Papers (...) 41 (1884), 21.

[15] H. S. Wilf, A circle-of-lights algorithm for the "money-changing problem", Amer. Math. Monthly 85 (1978), 562-565.

INSTITUTE OF MATHEMATICS AGH ACADEMY OF MINING AND METALLURGY MICKIEWICZA 30 30-059 KRAK/OW, POLAND

\author{
INSTITUTE OF COMPUTER SCIENCE \\ JAGIELLONIAN UNIVERSITY \\ NAWOJKI 11 \\ 30-072 KRAK/OW, POLAND
}

Received on 20.11.1992

and in revised form on 25.3.1993 